Research Journal of Applied Sciences, Engineering and Technology 6(14): 2579-2583, 2013

DOI:10.19026/rjaset.6.3741

ISSN: 2040-7459; e-ISSN: 2040-7467

(C) 2013 Maxwell Scientific Publication Corp.

Submitted: December 26, $2012 \quad$ Accepted: January 23, $2013 \quad$ Published: August 10, 2013

Research Article

\title{
Based on the GIS of Urban Landscape Ecology Suitability Evaluation and Sensitivity Analysis
}

\author{
Liu Tiedong \\ Northeast Forestry University Faculty of Landscape Architecture, Heilongjiang Harbin, China
}

\begin{abstract}
The aim of this study is to value the urban garden land ecological suitability to understand the city landscape ecology problems. In view of the Dong Liao of Jilin are being degraded ecological system, using the high accuracy of terrain data and environmental survey data and GIS technology support, taking vector data structure model, the evaluation factor layer stack formation evaluation unit and using the factor analysis method to determine evaluation factor weight. By applying ArcMap space analysis software of different factor given weight stack of urban landscape ecology suitability evaluation and different factor of urban landscape ecology environmental impact sensitivity analysis. Reasonable planning for urban garden land and effectively protect the city's ecological environment provide scientific basis.
\end{abstract}

Keywords: Ecological suitability, GIS, sensitivity, urban landscape

\section{INTRODUCTION}

Garden green space suitability is defined as a town green infrastructure garden green land relative town natural ecological pattern and town other facilities construction pattern and the social development of civilization to green space requirement conditions of appropriate degree (Osgood and Suci, 1955). For a particular garden green land scale and spatial pattern, suitability is high, the mean in this space mode and the scale of development planning, can play the biggest garden green space comprehensive benefits (Champ, 1976; San, 2011). In our country in the process of rapid urbanization, urban blind expansion the waste of land resources, but also cause the city's ecological environment destruction (Zule et al., 1973; Vafeidis, 2010). Science, effectively protect the city's ecological environment and realizing urban rapid and healthy development, it has become one of the major issue which people pay more and more attention (Kane, 1981; Kok et al., 2007).

The city is social, economic and natural compound ecosystem, as urban compound ecosystem natural subject, urban green space is the maintenance of human survival and the ecological balance and urban residents enjoy the natural ecosystem service basic guarantee; In the process of rapid urbanization, urban green space has constantly been city building opaque surface instead of green space with citizens rising demand, the contradiction between the increasing urban garden city as the main natural landscape elements in alleviating these contradictions plays an important role mouth (Farès, 2007; Chen and Shi, 2010). Urban landscape green space quantity and quality is often used to evaluate influence and become an important indicator of the residence people choose. Therefore, the urban landscape green space of scientific evaluation and reasonable planning is very important (Andreas, 2001). Urban level garden green space though various types, size differ, but in garden city standard can't comprehensive evaluation of the ecological landscape benefits, social benefits and economic benefits. Therefore, it is necessary to establish a set of scientific and reasonable comprehensive evaluation index system of urban landscape green space suitability evaluation for urban landscape green space system planning and construction to provide scientific basis.

In recent years, experts and scholars in all kinds of urban landscape ecology suitability evaluation on a lot of research, researches focus on the method, model and applied research and basic theoretical research. Method and main model of fuzzy comprehensive evaluation method, artificial neural network, genetic algorithm, the grey relation projection model and so on Romero (2004) and Goyal (2010).

The objective of this study is to understand the city landscape ecology problems, effective evaluation of urban garden land ecological suitability, taking Dong Liao landscape ecology system for example, the use of high precision of terrain data and environmental survey data, based on the GIS technology will evaluation factor layer stack formation evaluation unit and using the factor analysis method to determine the weight of evaluation factors, different factor given weight stack of urban landscape ecology suitability evaluation and other sensitive factor research, summarizes the problems and put forward the corresponding countermeasures, for urban landscape ecology system 
planning and provide scientific basis for decisionmaking.

\section{ECOLOGICAL SUITABILITY AND SENSITIVITY EVALUATION BASED ON GIS}

Select urban landscape ecology evaluation factors: Topography and surface coverage type is affected the city landscape ecology system of the two basic factors. They not only control the soil development, hydrological condition of differentiation, but also influence the occurrence of soil and water loss, decided to land use space differentiation. Vegetation cover type is land ecological system the external performance, its abundant biological production function is the system has the size of the mark. Vegetation and land ecological system in the process of all has a direct or indirect relationship, the typical environmental protection and control function. In order to protect the mountain natural vegetation, maintenance and optimization of its original habitat factors from alien species and the influence of human activity, from the influence of the factors of natural vegetation landscape, through the suitability evaluation to identify the study area development and protection of natural forest area, as the key to study the foundation of ecological optimization pattern.

Comprehensive consideration of the Dong Liao city development goals and urban land use current situation and the current problems in urban construction, the selection of the water, land use status, slope, geology, geomorphology, reserve factors as evaluation factors, waters include rivers, lakes and reservoirs and other surface waters in improving urban landscape quality, improve the city space environment, adjust the urban temperature humidity, maintain normal water cycle, etc. and at the same time, plays an important role in the waters near the ecological environment has great influence on the development of urban land, in principle, should as far as possible away from the water to water so as to avoid the destruction of ecosystem and water pollution. The existing land use mode determines urban landscape ecological suitability. Terrain is influence on the land development and construction of one of the limiting factors, too steep slope is easy to cause the various geological disasters, generally not suitable for urban landscape green space planning. Nature reserves, scenic spots, forest park Dong Liao natural condition is superior has rich biodiversity system many types of plants and animals, nature reserves, scenic spot and the forest park should be protected prohibited development and construction.
Determining the evaluation unit: Based on the evaluation factors, in order to protect and develop large garden green space as the basic goal, the suitability of the basic principles mainly consider the following:

- The development of high priority (i.e., high suitability or lower resistance value)

- with the increase of the slope, the priority of development has increased

- Evergreen broad-leaved forest and simao pine forest as the main protection object, so the existing forest land should be as development evergreen broad-leaved forest and pine wood preferred location

- The vegetation types and key protection types of vegetation characteristics more close, the species motion resistance is small

- Gentle slope cultivated land, especially the low altitude area, the gentle slope of the cultivated land should be protected as the basic farmland, 6 influence by human land types, its development has great value of resistance. First based on the evaluation factors and grading standards, based on the research of Digital Elevation Model (DEM), which generate landscape level hierarchical graph and grade classification diagram; Based on the above evaluation of the basic principle of in front of the evaluation factor layer, grade assessment score.

Establish spatial attribute database: This study, in accordance with the urban landscape of land ecological suitability degree it is divided into five levels: the most appropriate, is suitable, basic suitable, not suitable for and not land. Will not suitable for land and not land belong to basic ecological control area, in this area to ban all construction activities, to ensure that the ecological security; Will the most suitable land and is suitable to land into construction control area, land for construction purposes generally can't surpass this area; The basic appropriate land belong to ecological buffer area, used in the basic ecological control area and construction control area isolation, to ensure that they are a buffer space. Each objective is corresponding to a group of factors, geographic information coding model is to attribute the repository geographic information and knowledge base of geographic information undertake unity coding model, of which each factor can be compiled for a code. For each evaluation factor are divided into different types of indicators. Such as Table 1.

Table 1: The classification of residential elements

\begin{tabular}{llllll} 
Score & $91-100$ & $71-90$ & $51-70$ & $31-50$ & $0-30$ \\
\hline Evaluation classification & Actor & Good & Can be & Difference & Inferior \\
Density & $0.26-0.3$ & $0.21-0.2$ & $0.16-0.20$ & $0.11-0.15$ & $<0.10$ \\
Residential & $>0.2$ & $0.15-0.2$ & $0.10-0.15$ & $0.05-0.10$ & $<0.05$ \\
Level & $>10.0$ & $10.00-6.0$ & $6.00-5.00$ & $5.00-4.04$ & $<4.00$ \\
\hline
\end{tabular}




\section{APPLICATION}

Data sources: Data from the description of natural, social status words, maps and other data; 1: 10000 topographic map, as shown in Fig. 1; Vegetation pattern. Terrain data: 1:1000 based on the data of topographic map, the RTK surveying and mapping field, draw 1 to electronic topographic map and converted into GIS available data format. Using artificial interaction and supervised classification methods which patch information and the plaque attribute assignment, the establishment of terrain prototype model and image between direct and indirect relations.

Factor analysis method to determine the weight: If there are $n$ index, each sample has $\mathrm{p}$ variable, $\left(\mathrm{x}_{1}\right.$, $\mathrm{x}_{2}, \mathrm{~L}, \mathrm{x}_{\mathrm{p}}$ ), thus constitutes an order $\mathrm{n} \times \mathrm{p}$ matrix:

$$
X=\left[\begin{array}{cccc}
x_{11} & x_{12} & \mathrm{~K} & x_{1 p} \\
x_{21} & x_{22} & \mathrm{~K} & x_{2 p} \\
\mathrm{M} & \mathrm{M} & & \mathrm{M} \\
x_{n 1} & x_{n 2} & \mathrm{~K} & x_{n p}
\end{array}\right]
$$

In general, the original data with different dimensions or volume level, in order to ensure the reliability of the analysis results, the need for variable dimensionless normalization. The standardization and dimension reduction, $\mathrm{p}$ variables can be integrated into a $m$ new index, the variable can be expressed by the linear: $F_{1}, F_{2} L, F_{m}$, the variable $X$ can be expressed by the $\mathrm{F}_{\mathrm{m}}$ linear:

$$
X=L F+\varepsilon
$$

Type of:

$$
\begin{aligned}
F & =\left(F_{1}, F_{2} \mathrm{~L}, F_{m}\right) \\
L & =\left[\begin{array}{cccc}
l_{11} & l_{12} & \mathrm{~K} & l_{1 p} \\
l_{21} & l_{22} & \mathrm{~K} & l_{2 p} \\
\mathrm{M} & \mathrm{M} & & \mathrm{M} \\
l_{n 1} & l_{n 2} & \mathrm{~K} & l_{n p}
\end{array}\right]
\end{aligned}
$$

Optimal sample: $\mathrm{X}_{0}=\left(\mathrm{x}_{01}, \mathrm{x}_{02}, \mathrm{~L}, \mathrm{x}_{0 \mathrm{p}}\right)^{\mathrm{T}}$ can be by the formula structure:

$$
\begin{aligned}
& L=\left[\begin{array}{rrrr}
\Delta_{11} & \Delta_{12} & \mathrm{~K} & \Delta_{1 p} \\
\Delta_{21} & \Delta_{22} & \mathrm{~K} & \Delta_{2 p} \\
\mathrm{M} & \mathrm{M} & & \mathrm{M} \\
\Delta_{n 1} & \Delta_{n 2} & \mathrm{~K} & \Delta_{n p}
\end{array}\right] \\
& X_{o j}=\max _{1 \leq i \leq n}\left\{x_{i j}\right\}, j=1,2,3, \mathrm{~L}, p
\end{aligned}
$$
follows:

On the absolute difference matrix transform data as



Fig. 1: Original data

$$
\xi_{i j}=\frac{\min _{1 \leq i \leq n}\left\{x_{i j}\right\}+\rho \max _{1 \leq i \leq n}\left\{\Delta_{i j}\right\}}{\Delta_{i j}+\rho \max _{1 \leq i \leq n}\left\{\Delta_{i j}\right\}}
$$

The sample $X_{\mathrm{i}}$ and the optimal sample correlation degree $r_{i}$ :

$$
r_{i}=\frac{1}{p} \sum_{j=1}^{p} \xi_{i j}, \mathrm{i}=1,2,3, \mathrm{~L}, \mathrm{n}
$$

First of all, to determine the evaluation factor set:

$$
U=\left\{u_{1}, u_{2}, u_{3}, \mathrm{~L}, u_{n}\right\}
$$

Then determine the evaluation set: $\mathrm{V}=\left\{\mathrm{v}_{1}, \mathrm{v}_{2}, \mathrm{x}_{3}, \mathrm{~L}\right.$, $\left.\mathrm{v}_{\mathrm{n}}\right\}$. As the membership function with specific problems, combined with the actual situation, for the qualitative, quantitative factors take scoring system (Thierry de et al., 2007):

$$
v_{1}(x)=\left\{\begin{array}{lc}
1-\frac{x}{2 a_{1}} & x \leq a_{1} \\
\frac{a_{1}+a_{2}-2 x}{2\left(a_{2}-a_{1}\right)} & a<x \leq \frac{a_{1}+a_{2}}{2}
\end{array}\right.
$$$$
v_{2}(x)= \begin{cases}\frac{x}{2 a_{1}} & x \leq a_{1} \\ 1-\frac{a_{1}+a_{2}-2 x}{2\left(a_{2}-a_{1}\right)} & a<x \leq \frac{a_{1}+a_{2}}{2} \\ 1-\frac{2 x-\left(a_{1}+a_{2}\right)}{2\left(a_{2}-a_{1}\right)} & \frac{a_{1}+a_{2}}{2}<x \leq a_{2} \\ \frac{a_{2}}{2 x} & x>a_{2}\end{cases}
$$

$$
v_{3}(x)= \begin{cases}0 & x \leq \frac{a_{1}+a_{2}}{2} \\ \frac{2 x-\left(a_{1}+a_{2}\right)}{2\left(a_{2}-a_{1}\right)} & \frac{a_{1}+a_{2}}{2}<x \leq a_{2} \\ 1-\frac{a_{2}}{2 x} & x>a_{2}\end{cases}
$$

Spatial overlay analysis: Realize tool for ArcGIS 9.2 weighted superposition spatial analysis model. First the 


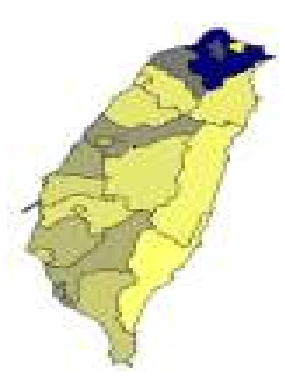

Fig. 2: Current land use map

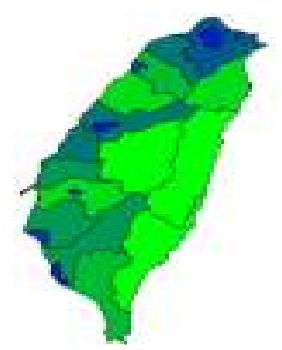

Fig. 3: Urban landscape green space distribution

grid data format of each evaluation factor analysis according to the grading standard results are heavy classification and then combined with the weight of each factor and calculates the value of ecological sensitivity comprehensive score. According to the superposition of the diagram to score, the factor of ecological sensitivity analysis is the use of GIS technology in the grid data attribute list add area attribute fields, calculate the sensitivity level area, then get the factor of ecological sensitivity data. Space stack effect such as Fig. 2 and 3.

\section{SUITABILITY EVALUATION AND SENSITIVITY ANALYSIS}

The city landscape ecology factor suitability evaluation: According to the urban landscape green space ecological suitability rating system (Table 1) the heavy classification, will not be suitable for land, not land belong to basic ecological control area, will be the most suitable land, the land is suitable to be divided into construction control area, the basic appropriate land as ecological buffer area. Using ArcGIS output classification results (Fig. 4), from Fig. 4 can see the model, the method to determine the basic ecological control area and the basic ecological line are consistent with each other, so the GIS model is practical and scientific. In the process of performance evaluation, in order to reflect the principle of ecological protection priority, the first use of ArcGIS software on ecological safety factors of basic farmland protection, nature reserves, protect water and humanistic landscape four indexes Mosaic operation get protected areas; Will these four for the offspring are weighted superposition operation. Evaluation scores Table 2.

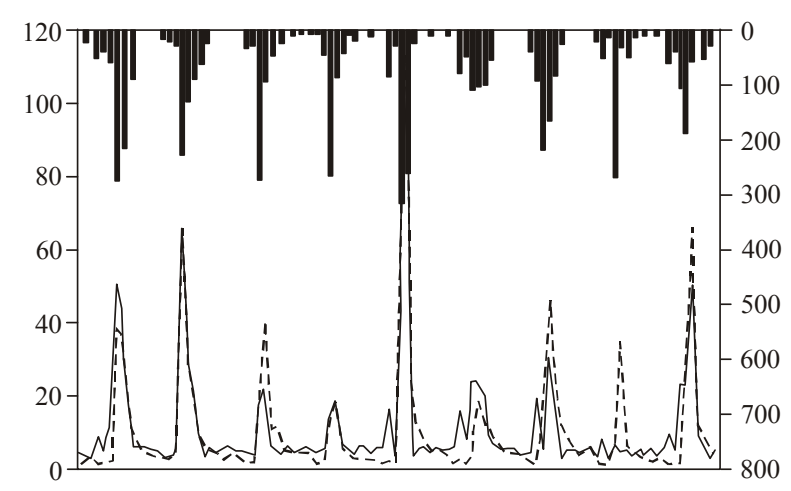

Fig. 4: Urban landscape ecological suitability assessment

\begin{tabular}{lllll}
\multicolumn{5}{l}{ Table 2: The listing after 3 years } \\
\hline & 0 & 1 & 2 & 3 \\
\hline Mean value & 18.520 & 17.410 & 12.620 & 10.670 \\
The median & 16.680 & 15.810 & 11.690 & 8.720 \\
Standard & 9.975 & 8.439 & 5.370 & 6.457 \\
Observation value & 209 & 209 & 209 & 209 \\
Significant level & & $* * *$ & $* * *$ & $* * *$ \\
\hline
\end{tabular}

The city landscape ecology sensitivity factor comprehensive evaluation analysis: The so-called ecological sensitivity is to point to in no loss or don't reduce the environmental quality between feeling distribution, for the prevention and control of the ecological environment and related regional policy formulation provides scientific basis. According to the research of ecological environment features, combined with the research focus and objective conditions, comprehensive analysis of many factors, choose elevation, gradient, slope direction, vegetation coverage, river water, land cover types six factors as Dong Liao urban landscape ecological sensitivity analysis of evaluation factors. To ecological factors on ecological environmental impact way and the degree of its classification, will be a single ecological factor sensitive degree is divided into 5 levels, in turn, is very sensitive area, highly sensitive area, moderate sensitive area, light sensitive area and not sensitive area Table 3.

Don't sensitive is mainly refers to the ecological environment basic stability, in the natural conditions and biological activities of the interference not prone to ecological environment problems. Don't sensitive area accounts for $4.14 \%$ of the total area, mainly distributed in the central valley and a flat area, frequent human activities. Light sensitive is mainly refers to the ecological environment basically stable, but in the natural conditions and biological activities of the interference will appear under mild ecological environmental problems. Light sensitive area accounts for $2.87 \%$ of the total area, the area is the smallest, mainly distributed in the relief is not big and vegetation coverage, human activities more frequent area. Moderate sensitivity refers to the ecological environment is stable, but in the natural and human 
Res. J. Appl. Sci. Eng. Technol., 6(14): 2579-2583, 2013

Table 3: Single element sensitivity classification evaluation result

\begin{tabular}{llllll}
\hline Evaluation factors & Not sensitive & Light sensitive & Moderately sensitive & Highly sensitive & Extremely sensitive \\
\hline Elevation & 11.28 & 13.46 & 16.54 & 20.01 & 40.89 \\
Slope & 9.75 & 14.05 & 20.83 & 23.53 & 60.21 \\
Vegetation coverage & 34.65 & 36.01 & 49.32 & 41.53 & 55.43 \\
River waters & 30.21 & 44.14 & 39.01 & 23.81 & 47.26 \\
Land cover types & 14.78 & 50.12 & 20.81 & 29.05 & 64.11 \\
\hline
\end{tabular}

action may destroy the original ecological environment, resulting in large degree of ecological environment problems. Moderate sensitive area accounts for $36 \%$ of the total area, in addition to the southern mountain area. No distribution widely distributed in the central valley. And the eastern mountain slope in the relatively concentrated distribution. These areas are relatively low altitude, slope vegetation is rich, larger. Highly sensitive, extremely sensitive is refers to the fragile ecological environment, in the natural and man-made easy appear under the action of ecological environment problems or has appeared the ecological environment problem.

\section{CONCLUSION}

Compared with the traditional numerical evaluation method, based on the GIS of urban landscape ecology suitability evaluation method will ground information acquisition, numerical calculation and spatial data processing organic combination, can be simple, intuitive, convenient and fast to quantitative analysis. This research in ecological investigation, on the basis of comprehensive consideration of the influence of urban construction land of natural, social and ecological factors, follow the "ecological priority," the principle of selecting evaluation index. Using factor analysis method to determine the weight of every evaluation index, reduced the weight evaluation of subjectivity. Using multi-factor comprehensive evaluation model of urban landscape ecology suitability evaluation, on the basis of the actual situation into basic ecological control area and construction control area, from the protection of urban ecological security point of view, urban land use Suggestions to urban landscape ecology environment of scientific evaluation and urban development in the future is of great significance.

By the use of GIS technology in the research of ecological environment problems, the selection of evaluation factors, on the ecological sensitivity, a comprehensive appraisal is made on the results of the study can be used as ecological planning design, ecological environment protection and urban ecological construction reference. The results of the evaluation of ecological sensitivity, on the one hand, largely depends on the level of evaluation data, on the other hand depends on the evaluation method. Therefore, in the future research work to carry out all-round, accurate, targeted urban landscape ecology environment survey and establish a rational urban landscape ecological suitability evaluation method system, in order to better serve the current ecological environment problems faced by the service.

\section{ACKNOWLEDGMENT}

The work was supported by "the Fundamental Research Funds for the Central Universities" Project number: DL12BA15.

\section{REFERENCES}

Andreas, M., 2001. Three-dimensional modeling and visualization of vegetation for landscape simulation. Landscape Urban Plann., 54: 5-17.

Champ, P., 1976. Evaluating english landscapes-some recent developments. Env. Plann., 18: 79-82.

Chen, H.F. and Z. Shi, 2010. Comprehensive evaluation of tobacco ecological suitability of Henan Province based on GIS. Agr.Sci.China, 10(16): 29-33.

Farès, B., 2007. Terrain modeling: A constrained fractal model. Proceeding of the 5th International Conference on Computer Graphics, Virtual Reality, Visualisation and Interaction in Africa, New York, pp: 197-204.

Goyal, S.K., 2010. GIS based spatial distribution mapping and suitability evaluation of groundwater quality for domestic and agricultural purpose in Kaithal district. India Env. Earth Sci., 12(9): 28-34.

Kane, P.S., 1981. Assecssing landscape attractiveness: A comparativelest of two new methods. Appl. Geograph., 1: 79-86.

Kok, L., S. Kreijkamp-Kaspers, D.E. Grobbee, E.H. de Haan, A. Aleman and Y.T. van der Schouw, 2007. Dietary phytoestrogen intake and cognitive function in older women. J. Gerontol. A Biol. Sci. Med. Sci., 62(5): 556-562.

Osgood, C.E. and G.J. Suci, 1955. Factor analysis of meaning. J. Exp. Psychol., 50(5): 325-338.

Romero, B.L., 2004. Spatial access to health care in Costa Rica and its equity: A GIS-based study. Soc. Sci. Med., 58: 1271-1284.

San, D., 2011. Methodology for quantifying groundwater abstractions for agriculture via remote sensing and GIS. Water Resour. Manag., 22(13): 45-52.

Thierry de, K., K. Marcvan and L. Maarten, 2007. Generating realistic terrains with higher-order Delaunay triangulations. Comp. Geomet., 36(1): 52-65.

Vafeidis, A.T., 2010. A proposed method for modelling the hydrologic response of catchments to burning with the use of remote sensing and GIS. Catena, 8(10): 34-40.

Zule, E.H., T. Anderson and D. Pitt, 1973. Measuring the landscape perceptual responses and physical dimension. Landscapes Res. News, 1(6): 1-5. 